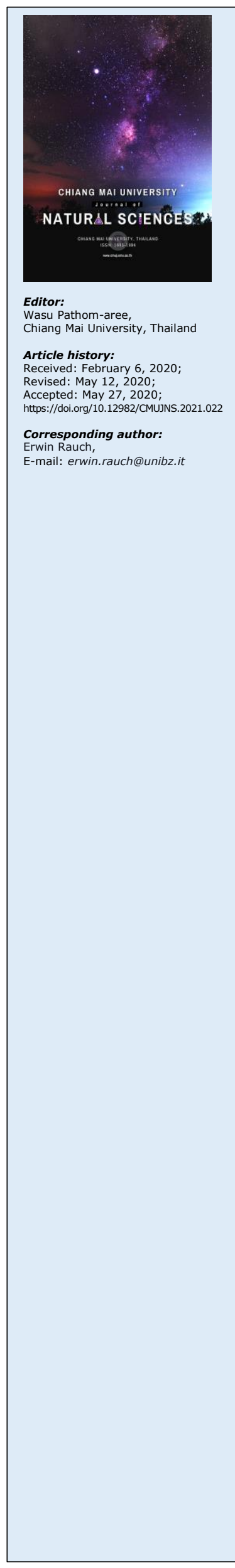

\title{
A Literature Review
}

\section{Research Fields and Challenges to implement Cyber- Physical Production Systems in SMEs: A Literature Review}

\author{
Rafael Rojas ${ }^{1}$, Erwin Rauch ${ }^{1, *}$ and Dominik T. Matt ${ }^{1,2}$
}

1 Industrial Engineering and Automation (IEA), Faculty Science and Technology, Free University of Bolzano, Bolzano 39100, Italy 2 Innovation Engineering Center (IEC), Fraunhofer Italia Research s.c.a.r.I., Bolzano 39100, Italy

Abstract Cyber-Physical Production Systems (CPPS) consists of the orchestration of single intelligent and connected cyber-physical systems (CPS) in order to perform what we call smart manufacturing. CPS collaborate in an intelligent way in order to obtain and maintain the optimum of the manufacturing process, handle disturbances and adapt to changing conditions. It might not be easy for small and medium-sized enterprises (SMEs) to implement such production system architectures in their shop floor. In this paper, we want to investigate existing scientific literature through a systematic literature review in order to identify the main research fields for implementing CPPS in smart SME factories. As a result, the identified research fields are critically discussed, highlighting those fields that can be identified as the most difficult challenges for SMEs in the near future and giving directions for future research activities.

Keywords: Cyber-physical production systems, Cyber-physical systems, Industry 4.0, Internet of things, Smart manufacturing, Systematic literature review

Funding: This research has received funding from the European Union's Horizon 2020 research and innovation program under the Marie Skłodowska-Curie grant agreement No 734713.

Citation: Rojas, R., Rauch, E., and Matt, D.T. 2021. Research fields and challenges to implement cyber-physical production systems in SMEs: a literature review. CMUJ. Nat. Sci. 20(2): e2021022. 


\section{INTRODUCTION}

With the trend of Industry 4.0 and digitalisation traditional shop floors will become capable to communicate with their machines, with their products and with their environment sending their status and their availability to CPPS. The increase of processing capacity of modern embedded systems and developments in communication systems increased the capabilities of CPPS from processing and transmitting acquired data towards an intimate interaction with physical processes and other elements in the production system (Lee, 2008). The processing capabilities and their connectedness make CPS able to exchange more structured information about their own perception of their environment (environment-awareness) and their self-state (self-awareness) in order to make intelligent decisions autonomously. When objects in a manufacturing system have cyber-physical capabilities and are connected to the same network, the possibilities for system automation are extended beyond the limits of traditional feedback control systems. In such a case it will become possible to create a body of collaborative autonomous $\mathrm{CPS}^{\prime}$ that regulates themselves thanks to the capacity of each single entity to retrieve information from its peers and itself. This kind of control systems is called a networked control system (Rachana, 2010). The practical use of such systems in factories is impeded by shortcomings like the lack of common standards for both horizontal and vertical integration, models for controlling complex structures, and qualified personnel (Cherubini et al., 2016). Especially, SMEs are often in difficulties with a comprehensive adoption of the concepts of Industry 4.0 and CPPS (Egger et al., 2017).

The aim of this work is, therefore, to identify past and current research fields for implementing CPPS in industry through a detailed literature review and to discuss afterwards, which of these research fields might present the most difficult challenges for SMEs in introducing Industry 4.0 and CPPS.

\section{MATERIALS AND METHODS}

The authors applied systematic literature review (SLR) as methodology in this study because it is based on a systematic, method-driven and replicable approach (Booth et al., 2016). According to Tranfield et al. (2003), SLR is characterised by a scientific and transparent process that aims to minimise bias through exhaustive literature searches and by providing an audit trail of the reviewer's procedures. Compared to other instruments, like co-citation analysis, SLR takes into account every source beyond the number of citations, which naturally are relatively low for recently published works.

As research on CPPS is relatively new and an emerging field, this factor played an important role in the selection of the research methodology for our study. In our study, we applied the procedure of SLR described by Denyer and Tranfield (2009) following the four steps: 1) establishing the research objectives; 2) defining the conceptual boundaries of the research; 3 ): setting out the data collection by defining the inclusion/exclusion criteria and 4) reporting the validation efforts.

The objectives of our literature review are to analyse the current state of the art regarding CPPS, with special focus on past and current research fields. The conceptual boundaries of this search were to use the term 'cyber-physical' combined with the term 'production system' and their variants of combination. To conduct the literature search the research team defined also the inclusion and exclusion criteria such as database, search period or search terms to cite only the main important ones. A full overview of the complete inclusion and exclusion criteria is given in Table 1. 
Table 1. Inclusion and exclusion criteria and related search count (see also Rojas and Rauch, 2019).

\begin{tabular}{|c|c|c|}
\hline Limitation & Inclusion / Exclusion criteria & Count \\
\hline Source & Scopus & - \\
\hline Search in & Article title, Abstract, Keywords & - \\
\hline $\begin{array}{l}\text { Search terms } \\
\text { and connections }\end{array}$ & $\begin{array}{l}\text { "Cyberphysical production system" OR "Cyber-physical production } \\
\text { system" OR "Cyber physical production system" OR "Cyberphysical } \\
\text { manufacturing system" OR "Cyber-physical manufacturing system" OR } \\
\text { "Cyber physical manufacturing system" OR "Cyberphysical assembly } \\
\text { system" OR "Cyber-physical assembly system" OR "Cyber physical } \\
\text { assembly system" }\end{array}$ & 1280 \\
\hline Time & $2012-2017$ & 1233 \\
\hline Source type & $\begin{array}{l}\text { Conference proceedings, journal contribution (excluding book chapters, } \\
\text { books, editorials, short surveys, trade journals) }\end{array}$ & 1035 \\
\hline Language & English & 975 \\
\hline \multirow[t]{2}{*}{ Screening } & First phase of coding: examination of title and abstract & 310 \\
\hline & Second phase of coding: examination of the whole paper & 165 \\
\hline
\end{tabular}

An appropriate validation of the results is crucial for an SLR. The coding scheme used in this case is based on two phases. In a first phase title and abstract were used for the evaluation of their relevance of the study. The criteria for judging the relevance for this study were i) high relevance, ii) medium relevance and iii) low relevance. Only those works with a high relevance were used for the next phase. In a second phase the whole paper was examined. The coding has been conducted in parallel by two different persons. Were these persons not of the same opinion, a third person went through deciding the relevance for our study. The used criteria in this phase were the same as in the first phase.

In total, we examined 975 works, of which 810 were excluded due to the screening as described before. The remaining 165 works are representing a period of six years from 2012 to 2017 . The start year 2012 was selected as the term Industry 4.0 has been defined in 2011 (at the Hannover fair) and no works have been published in 2011. The end year 2017 has been selected as the dataset was collected at the end of 2018. Most of the research identified is based on conference papers $(67 \%)$, while publications in journals showed a lower value (33\%). Such a high rate of conference papers is an indicator that the field is in a developing stage and the results are beginning to be consolidated at the journal level. Rojas and Rauch (2019) use the same literature review dataset to develop a CPPS framework as well as a control system architecture. In this paper, the authors emphasise more on the identification of research fields and future challenges for SMEs in implementing CPPS.

\section{RESULTS}

In order to better understand the identified literature, we have carried out a detailed content analysis reading each of the identified scientific works. Based on this approach, we have identified the following six main research fields (RF) for implementing CPPS in a company (Table 2 ): 
Table 2. Identified research fields for implementing CPPS.

\begin{tabular}{|c|c|}
\hline Research Fields (RF) & Short Description \\
\hline $\begin{array}{l}\text { 1) Multi-agent systems } \\
\text { and holonic } \\
\text { manufacturing }\end{array}$ & $\begin{array}{l}\text { Both holonic systems and multi-agent systems are conceptual enablers of } \\
\text { CPPS. This means that we have flexible and reconfigurable manufacturing } \\
\text { systems composed by CPS which are capable of negotiating and arriving at } \\
\text { agreements to coordinate smart actions. }\end{array}$ \\
\hline $\begin{array}{l}\text { 2) Integration and } \\
\text { interoperability }\end{array}$ & $\begin{array}{l}\text { This research field addresses the implementation of an IoT in a body of CPS to } \\
\text { achieve the pervasive availability of the information, addressing the issue of } \\
\text { the interoperability of data representation between different systems, the } \\
\text { network architectures and details on the communication infrastructure at the } \\
\text { physical and logical level. }\end{array}$ \\
\hline 3) Data Analysis & $\begin{array}{l}\text { This research field is of capital importance when analysing the large amounts } \\
\text { of unstructured and heterogeneous data produced by the pervasive availability } \\
\text { of information in CPPS. To deal with this, big data provide a family of } \\
\text { techniques for data collection and interpretation focused on providing } \\
\text { recommendations for decision-making. }\end{array}$ \\
\hline 4) Cloud Computing & $\begin{array}{l}\text { Thanks to the availability of the internet in CPPS shop floors, it will be possible } \\
\text { to retrieve information and resources from remote third parties. Unlike the } \\
\text { cluster of integration and interoperability that addresses connectivity in a wide } \\
\text { sense, the research field of cloud computing is specifically related to the } \\
\text { technologies used for providing services remotely through the internet }\end{array}$ \\
\hline $\begin{array}{l}\text { 5) Human-machine } \\
\text { interaction }\end{array}$ & $\begin{array}{l}\text { In Industry } 4.0 \text {, humans play a central role in the production systems and } \\
\text { processes. In such an anthropocentric production system, humans are in } \\
\text { synergy with robots and other machines, which increases their capabilities } \\
\text { through interactive and supporting tasks. This research field reviews the main } \\
\text { technologies which allow for the integration of humans into CPPS. }\end{array}$ \\
\hline 6) Cyber Security & $\begin{array}{l}\text { Due to the connectivity of CPPS with worker, material, machines, IT systems } \\
\text { and also external systems, cyber security is a significant research field, in } \\
\text { today's factory of the future. In this field, we collect several contributions } \\
\text { regarding analysis and implementation of cyber security in CPPS. }\end{array}$ \\
\hline
\end{tabular}

In the following sections, a detailed content analysis summarises the actual state of the art in each of the identified research fields and challenges. In the content analysis, we gave scientific journals a higher priority compared to other document types such as conference papers, which show ongoing and not just finished research. Where only a few journal papers were available, conference papers were considered in the detailed overview, giving priority to recent studies.

\section{RF-1: Multi-Agent Systems and Holonic Manufacturing in CPPS}

Thanks to pervasive computing, distributed computing systems are a dominant computational paradigm today (Weiss, 1999). This concept has been tackled by the idea of artificial intelligence, in that intelligent behaviours "emerge" from the interaction of many simple entities. Together, the concept of pervasive computing and "emergent" smart behaviours resonate with the idea of CPPS as a body of autonomous entities that interact in order to achieve global objectives smartly. Two main paradigms have been proposed to enable the smart behaviour of autonomous entities in production systems. On the one hand, multi-agent systems, where proposed in the field of AI, characterise such distributed computing systems. On the other hand, holonic manufacturing systems propose production systems constituted by autonomous entities that interact in different kinds of hierarchic or egalitarian relations to achieve similar objectives to CPPS. In this section, we summarise the main literature found on both paradigms and their convergence in CPPS (McFarlane, 1995; Bussmann, 1998).

RF-1.1: Multi-Agent Systems (MAS). An agent may be defined as a system that is situated in an environment that is capable of exerting autonomous actions in said environment in order to meet its design objectives (Wooldridge and Jennings, 
1995). Beyond the fact that agents do not necessarily have a physical part, we desire to underline some of the important features which CPS shares with agents in an MAS (Weiss, 1999): (i) they are self-aware and ambient-aware; (ii) they react timely to ambient variations; (iii) they exhibit goal-oriented behaviour and (iv) they interact with their peers. The leveraging of such characteristics, as proposed by, Vogel-Heuser et al. (2015), Ji et al. (2016) Monostori et al. (2016) and Müller et al. (2016), shows MAS technology as the main enabler of smart collective behaviour in CPPS. Leitao et al. (2016) discussed how MAS technology may enable CPPS with modularity, flexibility, robustness, configurability and responsiveness. A parallel between the abilities of MAS systems and the requirements of CPPS was drawn in Vogel-Heuser et al. (2015). In Penas et al. (2016), the concept of agents is also described as an ideal paradigm to enable CPS by merging mechatronic systems and IoT. In Ilsen et al. (2017), the MAS paradigm was used to develop self-organisation techniques capable of reducing energy consumption in CPPS. The organisation algorithm was developed in a simulator and focused on machine tool selection, where the highest flexibility in process planning regarding resource efficiency can be found.

RF-1.2: Convergence of MAS and HMS in CPPS. The idea of holonic systems was introduced in Koestler (1968) and implemented in manufacturing in the early 1990s (Mathews, 1995). In Tharumarajah (1996), there is a parallel between holonic systems and fractals; a holon is an autonomous and cooperative building block within a holonic system and they can cooperate in an equalitarian way with different relative influences in building decisions. Moreover, holons may be composed of other holons in a selfsimilar way, in the sense that a group of holons may also be considered a single holon. Parallelism between MAS and HMS was addressed early in McFarlane (1995) and Bussmann (1998). From these works, we can derive that both paradigms share the vision of being a manufacturing system with autonomous and cooperative units. Meanwhile, HMS is a conceptual paradigm motivated by the need to optimise manufacturing systems. MAS, with its origins in AI, provides methods to achieve smart emergent behaviour.

Wang and Haghighi (2016) conclude that agents and holons are complementary concepts inside a CPPS. They propose a CPS architecture that combines both paradigms, agents and holons, with the implementation of the concept of function blocks defined in the standard IEC 61499. For the digital part of each CPS, there is an agent responsible for high-level decision-making and coordination with other peers and function blocks that address low-level control functions. With the aforementioned combination, it is possible to identify CPS as holons. The authors propose that separate holons may replace the traditional constitutive elements of CPS and provide an architecture distinguished by three parts: a physical part, a control system and planning part and a scheduling and execution control part. Pujo et al. (2016) proposed a wireless network of holons and an instance of a CPPS identifying CPS identified as holons. In Quintanilla et al. (2016), a similar network of holons was proposed from the perspective of cloud computing.

\section{RF-2: Integration and Interoperability for CPPS}

The concept of integration may be understood as the process of ensuring that the interactions between entities in a system are those necessary to achieve specific objectives (ISO, 2002). In the context of CPPS, where the fundamental components are CPS, we can classify the interactions as physical or digital. As the physical layer of a CPS is governed by the software layer, integration in the context of CPPS is generally referred to as digital integration. As was remarked on in Rojas et al. (2017), such a type of integration needs to be implemented within the different layers of the OSI model (Zimmermann, 1980). In this section, we will use this model between upper and lower layers. In respect to the lower layers, the issues related to the integration of CPS are associated with real-time data delivery, the fault-tolerance of the network, the data delivery mechanism and network architectures. In the context of CPPS, we can find works addressing this topics such as Astarloa et al. (2015), Astarloa et al. (2016), Cammin et al. (2016), Garcá et al. (2016) and Puhm et al. (2016). An issue which spans almost all of the layers in the OSI model is part of the development of plug-and-play systems, which are addressed in Furmans et al. (2010), Schleipen et al. (2015) and Dai 
et al. (2016). In this section, we will focus on the concept of integration and interoperability in the upper layers of the OSI model.

The concept of integration of systems in the context of CPPS was explored in Schmidt et al. (2015) through a literature review. These works present a method for the quantification of the degree of integration and classification of the methodologies presented in the academic literature. Salvidar et al. (2015) presented a methodology to integrate CPS into the complete framework of Industry 4.0 and underlined the importance of modelling the component as a process within the system to achieve effective integration.

We may understand an Industrial IoT system as an integration architecture, which allows for us to share information seamlessly between every entity in the system. This is a challenging task because the digital representation of information varies and depends on the software and hardware used. In fact, the digital representation of an object in one system may be interpreted as a noisy sequence of bits by another system. At this point, we introduce the concept of interoperability, which is referred to as the ability of one system to process and use the information of another system (Vernadat, 2007). Further, we regard the problem of the integration of CPS as a problem of interoperability between digital systems.

We have identified different models of integration in our collected literature that differ in functionality and requirements. On the one hand, there is integration between the layers that make up the traditional automation pyramid, (i) vertical integration and (ii) horizontal integration, which refers to the integration among the different instances of the value chain of the product. Both flavours of integration are also addressed in the conceptual cluster of cloud computing, but, as we will see in the following sections, the same issue can be addressed from another perspective.

RF-2.1: Vertical Integration. The vertical integration of CPS into the IoT was analysed in Yoon and Suh (2016), where an architecture for a manufacturing service bus (MSB) variant was presented based on the classification of services in a CPPS. Implementation guidelines were given and exposed in a case study. The MSB was presented as a major concept in the integration of CPPS (MESA, 2008; Morariu et al., 2013), which was derived from the concept of enterprise service bus (ESB), a software middleware architecture that allows for transparent communication and resource sharing between disparate digital systems (Chappell, 2004).

Lee (2008) discussed the integration of smart robot systems into automotive industry operations by linking the cyber-physical capabilities of the mobile robots with MES. They described the modelling of robotic operations and the unstructured environment as building up an architecture for the vertical integration of the data representations between the MES layer and the physical machine layer (robots). Another vertical integration approach for location-based decision-making has been presented in Arrais et al. (2017), where mobile robots in an industrial system are integrated for the determination of information about the geometric location and volumetric information of the parts. Neubauer et al. (2017) developed the concept of business process management for vertical process integration of CPPS investigating the applicability of subject-oriented process management. They summarised their lessons learnt from two industrial application scenarios.

RF-2.2: Horizontal Integration. Tao et al. (2017) present a simulation-based validation model of horizontal integration of value chains for intelligent supply-demand matching. In Weichhart et al. (2016), the software requirements to handle complex enterprise integration problems arising in CPPS have been presented with a focus on dynamically changing systems.

RF-2.3: Service Oriented Architecture (SOA) and other issues. Both vertical and horizontal integration may be achieved in several ways, but the SOA paradigm is recognised as an enabler of CPPS (MESA, 2008; Sauer, 2014; Lin et al., 2015; Leitao et al., 2016) and a best practice for implementing general digital systems (Papazoglou et al., 2007; Vernadat, 2007). This paradigm consists of the organisation of the digital resources intended to be shared between disparate digital systems in self-contained, platform independent, dynamically discoverable, invokable and composable services 
(Georgakopoulos and Papazoglou, 2009). This concept has been explored in Puttonen et al. (2015), Dai et al. (2016), Quintanilla et al. (2016), Morgan and O'Donnell (2017) and Tao et al. (2017), . SOA refers to the upper layers of the OSI models and should be implemented after establishing solutions for other technical issues, such as data representation, semantics, syntax and ontology. The vast literature available on the topic in computer science has found an echo in the field of CPPS to solve the problem of interoperability from different perspectives, as shown in studies like Hoffmann et al. (2016), Ploennigs et al. (2016) and Thramboulidis and Christoulakis (2016).

\section{RF-3: Data Analysis}

The field of data analysis is comprised of several applications where the main goal is to retrieve information from raw data streams. This problem can arise in different situations, such as removing noise from a sensor data stream, recognising a human figure in a video stream or forecasting the maintenance of a machine. In the first example, it is possible to have an idea of the behaviour of the signal and to design a filter based on a predefined model. The others, on the contrary, are highly unstructured without models available. These problems have attracted more attention in the fourth industrial revolution due to the capacity for extracting useful information from very large streams of unstructured raw data that has arisen with the pervasive digitisation of industrial systems.

We have analytic models and simulation-based models. Different topics on modelling for simulation systems in CPPS are addressed in Galaske et al. (2015), Hehenberger et al. (2016), and Moon et al. (2016). Weckenmann and Hartmann (2015) and Schmitt et al. (2016) proposed a mathematical-physical to increase the quality of production in the context of cyber-physical systems. Geiger and Reinhart (2016) and Tao et al. (2017) exploited a simulation-based model of a CPPS to achieve supplydemand matching. Grundstein et al. (2017) used discrete-event simulation systems to benchmark an autonomous production control method which integrated all control tasks to meet the due dates. Freitag et al. (2015) explored resource sharing in a production network, while Kang et al. (2016) proposed a fault tolerance method in smart factories using a simulation-based framework for state estimation with feedback from massive CPS data. In Ilsen et al. (2017), simulation systems were used to achieve the reduction of energy consumption in CPPS. In the absence of models, the requirements of machine-learning algorithms in CPPS have been addressed in Maier (2014) and Niggemann and Frey (2015), using ML techniques for the automatic detection of anomalous and sub-optimal plant situations.

Windmann et al. (2015) and Mourtzis et al. (2016) have presented how the adoption of IoT in manufacturing, considering sensory systems and mobile devices will generate industrial Big Data. Gronau et al. (2016) presented the application of Big Data Analytics to extract the influence of the parameters of a CPPS on its behaviour. Marini and Bianchini (2016) described the techniques used to manage data volume and velocity during the data collection phase in a Big Data environment, and proposed data acquisition and analysis as a service. The implementation of CPPS in the chemical industry combining simulation and data mining has been presented in Ji et al. (2016).

\section{RF-4: Cloud Computing}

The concept of cloud manufacturing has evolved parallel to that of Industry 4.0, with Liu and Xu (2017) proposing a new paradigm to organise manufacturing resources over networks (Tao et al., 2011) based on new concepts in ICT and the internet. Among the concepts, that of the cloud may be understood as a digital distributed platform providing easily usable and accessible virtual resources such as hardware, data analysis platforms and services (Vaquero et al., 2008). Cloud manufacturing should enable access to manufacturing resources that can be rapidly provisioned and released with minimal management effort or service provider interaction ( $X u, 2012)$. Given such flexibility, cloud-related technologies have been proposed as the enablers of the fourth industrial revolution (Saldivar et al., 2015; Waitzinger et al., 2015; Wang et al., 2015; Quintanilla et al., 2016; Weinberger et al., 2016 ; Zhou et al., 2016). The interplay between Industry 4.0 and cloud manufacturing was explored in Liu and Xu (2017), where the differences of both concepts were explored. For the main difference, the authors found that CPPS relies on CPS platforms for vertical and horizontal integration. 
Meanwhile, cloud manufacturing was centred on cloud platforms for horizontal integration in order to connect and share manufacturing resources in the form of services. In Zhou et al. (2015) and Skarlat et al. (2016), cloud computing was catalogued as a key enabler of CPPS.

Following the literature, cloud manufacturing may be regarded from two perspectives. On the one hand, it is a complementary technology to CPPS that enables a gateway to integrate a body of CPS into the horizontal value chain (Schlechtendahl et al., 2015). This conceptualisation places cloud manufacturing at the crossroads between vertical and horizontal integration. The papers that fit in this category are Helo et al. (2014), Wang et al. (2016) and Tao et al. (2017), where the cloud-based integration of CPPS was discussed at the level of ERP systems, supply-demand matching and financial systems.

Other perspectives in the literature address cloud computing in manufacturing as a vertical integration tool using web-related technologies. Verl et al. (2012), Makarov et al. (2014), Yen et al. (2014), Zolotová et al. (2015), Caggiano et al. (2016), Ferreira et al. (2016), Quintanilla et al. (2016), and Tao et al. (2017) addressed the issue of connecting CPS inside a CPPS to make a cloud-based repository of services.

Both perspectives may present situations where real-time capabilities are need. This issue has been addressed in Makarov et al. (2014) and Mejás et al. (2017).

\section{RF-5: Human-Machine Interaction (HMI)}

During the third industrial revolution, we saw how robots and automation systems substituted humans in several tasks. However, in the logic of industry 4.0, CPS do not substitute humans, but work with them in a synergic way to support them through physical, sensorial and cognitive aid systems. Such a complementary system between humans and CPS has been addressed in Ansari and Seidenberg (2016), where the authors proposed a characterisation for optimal collaboration between them based on the degree of autonomy of CPS and the qualification of the humans for different tasks arising from the fourth industrial revolution. In fact, as commented on in the introduction, the fourth industrial revolution will exhibit new work areas where, as remarked in Becker and Stern (2016), Fantini et al. (2016) and Jiang et al. (2016), highly skilled occupations will increase, while low-skilled and auxiliary occupations will decrease. Humans will always exhibit a major degree of flexibility, more so than automated solutions. Consequently, the interaction between humans and CPS will remain central in industry 4.0 as Pirvu et al. (2016) discussed, by proposing such an interaction as the basic decomposition unit for the design of CPPS. This harmony may be possible only with the right information flow and interfaces between humans and machines/computers, commonly called user interfaces (UI). UIs will provide the means to configure CPS to perform fully automated tasks and to support humans in partially automated tasks.

To support humans, CPS need HMI methods, as explored in Gorecky et al. (2014) and Hold et al. (2016). In the aforementioned articles, we can appreciate how HMI methods have evolved from indicator lights, buttons and levers through to every-day Graphical User Interfaces (GUI), keyboards, mouse and touch-screens, before arriving to the concept of multimodal interfaces (Oviatt and Cohen, 2000), where the voice, hands, and entire body become a communication channel. We followed Gorecky et al. (2014) in order to identify the main research topics of HMI in the era of Industry 4.0.

RF-5.1: Automatic Speech Recognition. The field of automatic speech recognition (ASR), where the term voice user interface (VUI) is frequent, has a long history in computer sciences (OShaughnessy, 2008). Lotterbach and Peissner (2005) describe which aspects have to be considered before implementing VUIs in industrial environments. An interesting overview of the application of ASR in industrial maintenance was given in Goose et al. (2003). For example, the system Jennifer ${ }^{\mathrm{TM}}$ VoicePlus is a VUI developed by Lucas Systems oriented to warehouse logistics.

RF-5.2: Visual Gesture Recognition. By the term gestures, we refer to expressive and meaningful body motions, which span from the fingers, hands and arms through to face expressions with the intent of transmitting meaningful information or interacting with the environment. Several sensors can used to recognise gestures, and 
so the field of visual computing has been recognised as one of capital importance in Industry 4.0 (Posada et al., 2015). In particular, with the introduction of 3D imaging sensors, the possibilities for visual gesture recognition have grown (Sansoni et al., 2009). Gesture recognition has been long appreciated as a method for interacting with robots (Triesch and van der Malsburg, 1998; Brethes et al., 2004). An interesting review of the applications and technologies of this topic has been given in Mitra and Acharya (2007) and a gesture-based UI control for a factory loading station was evaluated in Heimonen et al. (2013).

RF-5.3: Enhanced Reality. Enhanced reality, or augmented reality (AR), is the augmentation of physical world information by digital information that is superimposed on a view of the physical world (Craig, 2013). Several technologies can be used to implement it on each of the human senses. Currently, it is primarily implemented in the visual medium using mobile devices, smart glasses, and spatially augmented reality (SAR), where virtual objects are rendered directly within or on the user's physical space (Raskar et al., 1998). In the technical report by Heutger and Kückelhaus (2014), cases of AR in logistics were commented on and, in Olwal et al. (2008), SAR was used to enhance HMI for industrial computer numeric controlled (CNC) machines.

RF-5.4: Physical Robot-Human interaction. Under the perspective of the multimodal interface, physical contact may be used to create an interaction interface for CPS. Such a concept has been extensively explored in robotics, in particular, the field of collaborative robotics (Haddadin et al., 2012), where the contact between humans as robot is expected to be frequent. The mechatronic design of a collaborative robot allows it to sense the qualities of physical contact, making possible to convey information through physical interaction. The introduction of such systems into industrial environments has been addressed in Guerin et al. (2015) and Cherubini et al. (2016). On the other hand, we have human-robot augmentation systems, where the human's mechanical capacities involving motion control and force are augmented with wearable robotic systems (Bergamasco and Herr, 2016). Such systems may be used to improve the worker's mobility (Louie et al., 2015), dexterity (Taylor et al., 1999), resistance to fatigue (Shields et al., 1997) and force (Bogue, 2015).

\section{RF-6: Cyber Security}

Security is a transversal issue in modern IT technologies. As such, it spans CPPS pervasively across all of its components. Moreover, it has been recognised in Cardenas et al. (2009), Choo (2011) and Wu et al. (2016) that the concept of security takes on a new dimension when referring to CPS. In CPPS, the issue of security is of high importance for the IT and OT domains and their dense net of interaction, but its implementation should not interfere with operational business processes. The intrinsic heterogeneity of communication technologies and data exchanges in CPPS are a fertile ground for weak links. In fact, it is recognised in Ullrich et al. (2016) and Chhetri et al. (2017) that one of the major drawbacks of implementing ITC technologies in manufacturing is the issue of cyber security. Several attacks in CPS have been reported by Slay and Miller (2007), Falliere et al. (2011) and in Lee et al. (2014) with negative consequences and the associated economic costs. These examples reveal that the issue of security in CPS is qualitatively different from traditional ICT in that not only information may be the scope of external aggression. In fact, in these examples, the physical infrastructure was the objective of the attacks, implying that security is tightly coupled with safety. From this, we can understand that, in CPPS, security differs from traditional ICT systems because (i) it is tightly coupled with safety and (ii) the cyber domain may be used to attack the physical domain, and vice versa. The novelty of the behaviour of these issues is that the most common security issues in CPPS are addressed with traditional ICT security practices (Chhetri et al., 2017), but the scientific community is beginning to propose several new perspectives (Yampolskiy et al., 2013). An approach to identify such new vulnerabilities may be found in DeSmit et al. (2016), while Ullrich et al. (2016)analysed how security can be addressed in the context of CPPS in order to close the gap between the different perspectives that are currently analysing the security issue in a parallel form, developing alike solutions with different terminologies. By using three examples, they argue how CPPS' interoperability, system 
design, and self-protection can actually benefit from common development. An overview of the issues associated with security when dealing with cloud manufacturing and remote control has been addressed in Buckholtz et al. (2016). In Yampolskiy et al. (2013), a classification of attacks on CPS was introduced with four categories based on the origin of the attack and the target (physical or cyber domain), while Chhetri et al. (2017) emphasised the practical applicability of cross-domain security analysis in CPPS. In the field of additive manufacturing, two interesting works have been published showing how it is possible to attack the physical domain from the cyber (Chhetri et al., 2016) and the other way around (Al Faruque et al., 2016). In fact, in Al Faruque et al. (2016), it was shown how the acoustic field (physical domain) produced by a 3D printer may be used to violate the privacy of the 3D model that is actually printed (virtual domain). In Chhetri et al. (2016), it was shown how the corruption of the data of 3D models (cyber domain) may induce the desired structural failures in printed structures (physical domain). These works show that a major concern in CPPS security analysis is the system's model where the physical, platform and software layers are coupled. Based on such an approach, control theory throws a new light on the issue of security. In Pasqualetti et al. (2013), an observation of the state of a system's model was used to detect and identify attacks on CPS. Cross-domain secure design was addressed in Zheng et al. (2016) (this addresses the control system) and, in Teixeira et al. (2012)from the control theory perspective. As in CPPS, the distributed control system is fundamental. It is also a key security issue. In Xu et al. (2005), the issues of collaborative control related to the distributed device networks under open and dynamic environments are addressed with traditional ICT methods. In Amin et al. (2009), the study addressed the problem of designing a network control that is robust to packet jamming.

\section{DISCUSSION}

On the basis of the research fields identified for the introduction of CPPS in industrial companies, we would now like to critically discuss which of these will pose the greatest challenges for small and medium-sized companies. For this discussion, we have developed a framework on the basis of the research fields found, which should visually illustrate the context of the individual research topics with the entire CPPS as well as their relation to each other. The framework is shown in Figure 1.

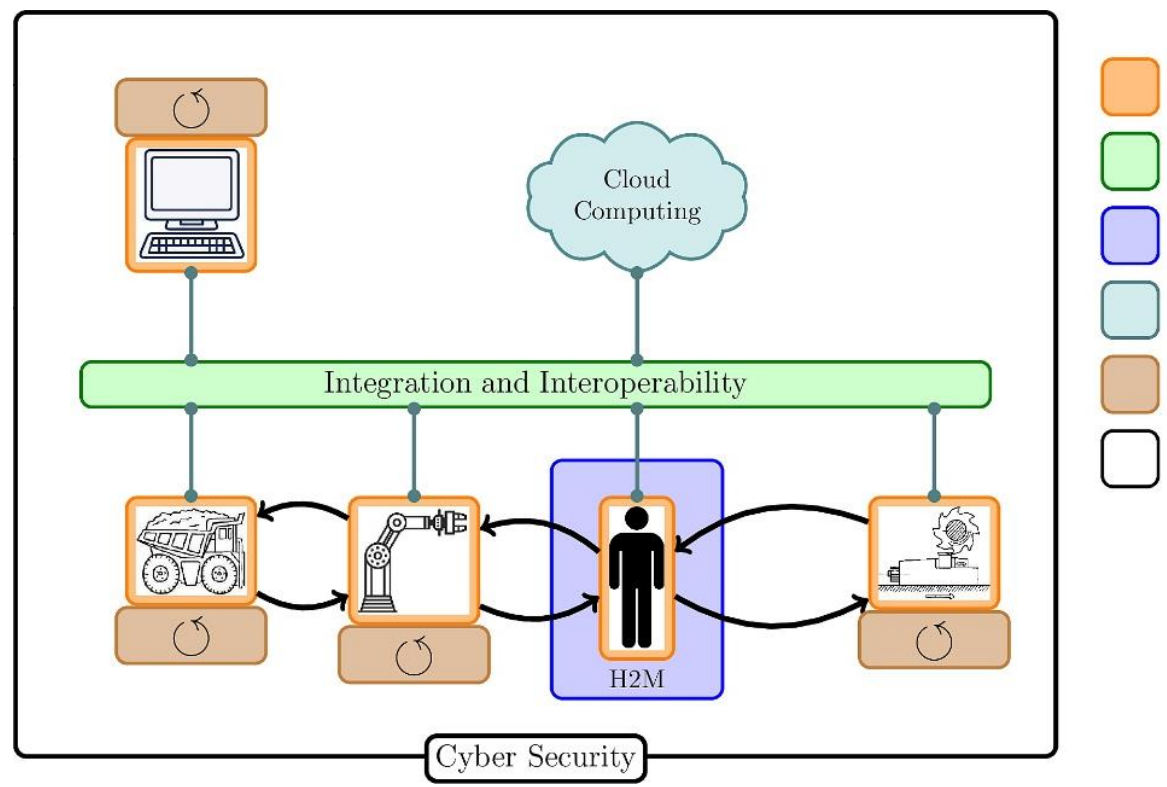

Multi Agent Systems and Holonic Manufacturing Systems Integration and Interoperability Human-Machine Interaction Cloud Computing

Data Analysis Cyber Security

Figure 1. Framework of research fields for the implementation of CPPS in SMEs (Rojas and Rauch, 2019). 
In our opinion, the connectivity of all elements in the CPPS is the basic prerequisite for smart manufacturing. It allows the manufacturing system to communicate and interact with all the CPS elements in the whole CPPS as well as with human operators and the environment outside of the manufacturing system. It is, therefore, essential for SMEs to overcome the hurdle of "integration and interoperability" creating a vital vein for retrieving information and for sharing information along this vein. According to the experience of the authors working together very closely with SME industry, they currently have difficulties of interoperability on an application level (software applications in the company that are not able to share information or to communicate with each other) as well as on the machine level (machine control systems that are stand-alone systems without the possibility to make them interact). As the knowledge of IT departments in typical SMEs is often not fully developed, they struggle with the practical realisation of a fully connected shop floor. As mentioned before, we see here a first big challenge for SMEs, where a collaboration with research institutions would help to set up first pilot-lines or cells.

Once the prerequisite for connectivity is given, multiple agents in the manufacturing system can be integrated into the system as "holons", where all the nodes must be defined as well as the mechanisms with which the individual elements interact with the other holons in the system. In this case, providers of machines in the manufacturing shop floor usually have singular solutions for orchestrating elements from the same brand with each other. Superordinate middleware solutions, able to manage different CPS elements equipped with a control from different providers, are currently not available on the market and have yet to be developed. Here we see a next big challenge for SMEs to develop and implement such systems, with which a production system consisting of very different holons can be efficiently orchestrated. In this case, SMEs are depending from the provider market as they usually do not have qualified developers of such systems in their own company. As providers want to enforce companies to buy machines from the same (their) brand, we do not expect a quick solution for this problem. Also, here SMEs should try to work together and directly with research institutions in order to build up a pilot line or cell for testing this technologies in practice.

A special "holon" in the manufacturing system are humans in the figure of operators or supervisors, they play a fundamental role in the smart factory of the future, but, at the same time, have to interact with much more smart CPS elements than in the past. The way for interaction in such a situation is that what we call a "human-machine interaction". Most of the machine providers offer well-designed solutions for humanmachine interfaces, like touchscreens, pendants, control units or other devices like smartphones or tablets. Therefore, we do not see that this research field will show a big challenge for SMEs in order to introduce and run a CPPS. Here the challenge is more how to improve aspects like ergonomics or usability, which is not a must have argument. Developments in this direction can be based on the applications of new technologies for interaction between man and machine-like Virtual Reality (VR) or Augmented Reality $(A R)$, as well as more intuitive and easy programming of robots or other machines.

"Cloud computing" represents the connection of the manufacturing system with its environment outside the shop floor. In recent years, many innovations were transferred from research into industry and also SMEs introduced cloud computing technologies in order to reduce the need of powerful hardware devices and IT infrastructures as well as to increase data security and availability in their company. The technologies around cloud computing are quite consolidated and have reached also smaller enterprises. Therefore, we do not see any bigger challenge for SMEs in this research field.

The capabilities of intelligent CPS on the shop floor will allow to decentralise functionalities for analysing data and doing decision-making autonomously based on the results of the analysis. Therefore, "data analysis" will become an important topic for integrating CPS in a superordinate CPPS network. Processing of real-time data of CPS will have an impact on the quality of production planning and control as well as on the maintenance and availability of machines and the entire system. Up to now, there are only few brand-neutral solutions on the market, which are adopted to the needs and resources of SMEs and which allow to analyse real data. In most of the cases, machine producers develop data analysis tools for their branded products, which again results in 
a high diversity on the market and, therefore, again in difficulties to match the data analysis results of different CPS in the systems. Therefore, we see again a big challenge for SMEs also in the implementation of data analysis tools and especially in an efficient use of this analyses carried out in isolated points of the manufacturing system.

In "cyber security" most of the SMEs operating on the market are not well prepared for cyber-attacks from outside or they dispose only about very basic measures to prevent such cases. However, since this is a problem that is faced by large and small companies alike and the necessary financial resources for development work in this direction can be immense, solutions will probably be developed from top to bottom. This means that large companies have to face this problem immediately because of their visibility and vulnerability, which will lead to the development of commercial solutions. Over time, these will be scaled to smaller companies and, thus, be financially accessible also for them. Until then, it can at least be assumed that SMEs will tend to be less affected or attractive for cyber-attacks than large companies. Therefore, despite the undiscussable importance of the problem itself, we do not see a big challenge for SMEs in a short-medium term.

\section{CONCLUSION}

In this paper, the focus was on current research fields and the resulting challenges for SMEs to introduce CPPS in production. The identification of the current research directions was methodically carried out by means of an SLR by filtering and analysing the current scientific literature. Within the SLR, six research fields were identified, which have to be considered with regard to the implementation of CPPS in companies: 1 ) multi-agent systems and holonic manufacturing, 2) integration and interoperability, 3) data analysis, 4) cloud computing, 5) human-machine interaction and 6) cyber-security.

A subsequent discussion and critical validation of the individual research fields revealed that the three research fields in particular representing a major challenge for SMEs are multi-agent systems and holonic manufacturing, connectivity and interoperability as well as data analysis. SMEs need simple solutions for connecting various systems and brands of devices in one CPPS as well as solutions to interpret the various decentralised data analysis results on a higher network level and to orchestrate all single and intelligent elements of the CPPS. There are currently no commercially available systems that offer such functionalities for SMEs. SMEs should, therefore, carry out research on these topics in form of collaborations with research institutions and case study research in their own pilot lines or cells.

Although the other research fields are also important, they often dispose of already consolidated technologies or sufficiently mature and commercially available systems. In other cases (cyber-security), SMEs are currently simply unable to cope with these challenges alone and depend here on the development of solutions for larger companies.

Despite the fact that CPPs are still a relevant topic in research, they have great potential and a major impact on the short- and medium-term design of production systems in industrial companies. CPPS are conceivable for any type of business scale, for both large companies and SMEs. While topics such as cloud computing and simple human-machine interaction can already be used today in SMEs, topics such as the integration and networking of holons within the manufacturing system as well as the analysis of production data pose a major challenge for many SMEs. In the future, further research will be needed to be able to scale systems that are initially often developed for large companies to SMEs.

As this research is part of a research project on introducing Industry 4.0 in SMEs, the author will use the results and findings of this work to address the next research activities in the project. This means that the research team will first test possible solutions for the above mentioned challenges in the form of laboratory case studies. In a second step, the research team will validate the proposed solutions practically in industrial case studies. 


\section{REFERENCES}

Al Faruque, M.A., Chhetri, S.R., Canedo, A., and Wan, J. 2016. Acoustic side-channel attacks on additive manufacturing systems. In Proceedings of the $7^{\text {th }}$ International Conference on Cyber-Physical Systems. IEEE Press. p. 19.

Amin, A., Cárdenas, A.A., and Sastry, S. 2009. Safe and secure networked control systems under denial-of-service attacks. Springer: In HSCC. 5469: 31-45.

Ansari, F., and Seidenberg, U. 2016. A portfolio for optimal collaboration of human and cyber physical production systems in problem-solving. International Association for Development of the Information Society.

Arrais, R., Oliveira, M., Toscano, C., and Veiga, G. 2017. A mobile robot based sensing approach for assessing spatial inconsistencies of a logistic system. Journal of Manufacturing Systems. 43: 129-138.

Astarloa, A., Moreira, N., Lázaro, J., Urbina, M., and Garcia, A. 2015. 1588-aware highavailability cyber-physical production systems. In 2015 IEEE International Symposium on Precision Clock Synchronization for Measurement, Control, and Communication (ISPCS). IEEE. p. 25-30.

Astarloa, A., Bidarte, U., Jiménez, J., Zuloaga, A., and Lázaro, J. 2016. Intelligent gateway for industry 4.0-compliant production. In Industrial Electronics Society, IECON 2016-42nd Annual Conference of the IEEE, pages 4902-4907. IEEE.

Becker, T., and Stern, H. 2016. Future trends in human work area design for cyberphysical production systems. Procedia CIRP. 57: 404-409.

Bergamasco, M., and Herr, H. 2016. Human-robot augmentation. In B. Siciliano and O. Khatib (eds), Springer Handbook of Robotics, chapter 70, pages 1875-1906. Springer.

Bogue, R. 2015. Robotic exoskeletons: a review of recent progress. Industrial Robot: An International Journal. 42: 5-10.

Booth, A., Sutton, A., and Papaioannou, D. 2016. Systematic approaches to a successful literature review. Thousand Oaks, CA: Sage Publications Ltd.

Brethes, L., Menezes, P., Lerasle, F., and Hayet, J. 2004. Face tracking and hand gesture recognition for human-robot interaction. In 2004 IEEE International Conference on Robotics and Automation ICRA'04. IEEE. 2: 1901-1906.

Buckholtz, B., Ragai, I., and Wang, L. 2016. Remote equipment security in cloud manufacturing systems. International Journal of Manufacturing Research. 11: 126-143.

Bussmann, S. 1998. An agent-oriented architecture for holonic manufacturing control. In Proceedings of first international workshop on IMS, Lausanne, Switzerland. p. $1-12$.

Caggiano, A., Segreto, T., and Teti, R. 2016. Cloud manufacturing framework for smart monitoring of machining. Procedia CIRP. 55: 248-253.

Cammin, C., Krush, D., Heynicke, R., Scholl, G., Schulze, C., Thiede, S., and Herrmann, C. 2016. Coexisting wireless sensor networks in cyber-physical production systems. In 21st International Conference on Emerging Technologies and Factory Automation (ETFA). IEEE, p. 1-4.

Cardenas, A., Amin, A., Sinopoli, B., Giani, A., Perrig, A., and Sastry, S. 2009. Challenges for securing cyber physical systems. In Workshop on future directions in cyber-physical systems security, page 5.

Chappell, D. 2004. Enterprise Service Bus. Sebastopol, CA: O'Reilly.

Cherubini, A., Passama, R., Crosnier, A., Lasnier, A., and Fraisse, P. 2016. Collaborative manufacturing with physical human-robot interaction. Robotics and ComputerIntegrated Manufacturing. 40: 1-13.

Chhetri, S.R., Canedo, A., and Al Faruque, M.A. 2016. Kcad: kinetic cyber-attack detection method for cyber-physical additive manufacturing systems. In 2016 IEEE/ACM International Conference on Computer-Aided Design (ICCAD). IEEE. p. $1-8$.

Chhetri, S.R., Wan, J., and Al Faruque, M.A. 2017. Cross-domain security of cyberphysical systems. In 2017 22nd Asia and South Pacific Design Automation Conference (ASP-DAC). IEEE. p. 200-205.

Choo, K.-K. R. 2011. The cyber threat landscape: Challenges and future research 
directions. Computers \& Security. 30: 719-731.

Craig, A.B. 2013. Understanding augmented reality: Concepts and applications. Burlington, MA: Morgan Kaufmann.

Dai, W., Huang, W., and Vyatkin, V. 2016. Enabling plug-and-play software components in industrial cyber-physical systems by adopting service-oriented architecture paradigm. In Industrial Electronics Society, IECON 2016-42nd Annual Conference of the IEEE. IEEE. p. 5253-5258.

Denyer, D., and Tranfield, D. 2009. Producing a systematic review. In D. A. Buchanan and A. Bryman (Eds.), The Sage handbook of organizational research methods (pp. 671-689). Thousand Oaks, CA: Sage Publications Ltd.

DeSmit, Z., Elhabashy, A.E., Wells, L.J., and Camelio, J.A. 2016. Cyber-physical vulnerability assessment in manufacturing systems. Procedia Manufacturing. 5: $1060-1074$.

Egger, G., Rauch, E., Matt, D.T., and Brown, C.A. 2017. (Re-) Design of a Demonstration Model for a Flexible and Decentralized Cyber-Physical Production System (CPPS). MATEC Web of Conference. 127: 01016.

Falliere, N., Murchu, L.O., and Chien, E. 2011. W32. stuxnet dossier. White paper, Symantec Corp., Security Response. 5.

Fantini, P., Tavola, G., Taisch, M., Barbosa, J., Leitao, P., Liu, Y., Sayed, M.S., and Lohse, N. 2016. Exploring the integration of the human as a flexibility factor in cps enabled manufacturing environments: methodology and results. In Industrial Electronics Society, IECON 2016-42nd Annual Conference of the IEEE. IEEE. p. 5711-5716.

Ferreira, F., Faria, J., Azevedo, A., and Marques, A.L. 2016. Product lifecycle management enabled by industry 4.0 technology. In Advances in Transdisciplinary Engineering. 3: 349-354.

Freitag, M., Becker, T., and Duffie, N.A. 2015. Dynamics of resource sharing in production networks. CIRP Annals-Manufacturing Technology. 64: 435-438.

Furmans, K., Schönung, F., and Gue, K.R. 2010. Plug and work material handling systems. Progress in Material Handling Research. 132-142.

Galaske, N., Tantik, E., and Anderl, E. 2015. Discrete-event simulation software for modeling flexibility-driven manufacturing processes. In ASME 2015 International Design Engineering Technical Conferences and Computers and Information in Engineering Conference, pages V004T05A015-V004T05A015. American Society of Mechanical Engineers.

Garcá, M.V., Irisarri, E., Pérez, F., Estévez, E., and Marcos, M. 2016. Opc-ua communications integration using a cpps architecture. In Ecuador Technical Chapters Meeting (ETCM). IEEE. 1: 1-6.

Geiger, F., and Reinhart, G. (2016). Knowledge-based machine scheduling under consideration of uncertainties in master data. Production Engineering. 10: 197207.

Georgakopoulos, D., and Papazoglou, P. 2009. Service-oriented computing. Cambridge, MA: MIT press.

Goose, S., Sudarsky, S., Zhang, X., and Navab, N. 2003. Speech-enabled augmented reality supporting mobile industrial maintenance. IEEE Pervasive Computing. 2: 65-70.

Gorecky, D., Schmitt, M., Loskyll, M., and Zühlke, D. 2014. Human-machine-interaction in the industry 4.0 era. In $201412^{\text {th }}$ IEEE International Conference on Industrial Informatics (INDIN). IEEE. p. 289-294.

Gronau, N., Grum, M., and Bender, B. 2016. Determining the optimal level of autonomy in cyber-physical production systems. In 2016 IEEE $14^{\text {th }}$ International Conference on Industrial Informatics (INDIN). IEEE. p. 1293-1299.

Grundstein, S., Freitag, M., and Scholz-Reiter, B. 2017. A new method for autonomous control of complex job shops-integrating order release, sequencing and capacity control to meet due dates. Journal of Manufacturing Systems. 42: 11-28.

Guerin, K.R., Lea, C., Paxton, C., and Hager, G.D. 2015. A framework for end-user instruction of a robot assistant for manufacturing. In 2015 IEEE International Conference on Robotics and Automation (ICRA). IEEE. p. 6167-6174.

Gupta, R.A., and Chow, M.-Y. 2010. Networked control system: Overview and research trends. IEEE Transactions on Industrial Electronics. 57: 2527-2535. 
Haddadin, S., Haddadin, S., Khoury, A., Rokahr, T., Parusel, S., Burgkart, R., Bicchi, A., and Albu-Schäffer, A. 2012. On making robots understand safety: Embedding injury knowledge into control. The International Journal of Robotics Research. 31: 1578-1602.

Hehenberger, P., Vogel-Heuser, B., Bradley, D., Eynard, B., Tomiyama, T., and Achiche, S. 2016. Design, modelling, simulation and integration of cyber physical systems: Methods and applications. Computers in Industry. 82: 273-289.

Heimonen, T., Hakulinen, J., Turunen, M., Jokinen, J.P.P., Keskinen, T., and Raisamo, R. 2013. Designing gesture-based control for factory automation. In INTERACT. p. 202-209.

Helo, P. Suorsa, M., Hao, Y., and Anussornnitisarn, P. 2014. Toward a cloud-based manufacturing execution system for distributed manufacturing. Computers in Industry. 65: 646-656.

Heutger, M., and Kückelhaus, M. 2014. Augmented reality in logistics. Technical report, DHL.

Hoffmann, M., Thomas, P., Schütz, D., Vogel-Heuser, B., Meisen, T., and Jeschke, S. 2016. Semantic integration of multi-agent systems using an opc ua information modeling approach. In 14th International Conference on Industrial Informatics (INDIN). IEEE. p. 744-747.

Hold, P., Ranz, F., Sihn, W., and Hummel, V. 2016. Planning operator support in cyberphysical assembly systems. IFAC-PapersOnLine. 49:60-65.

Ilsen, R., Meissner, H., and Aurich, J.C. 2017. Optimizing energy consumption in a decentralized manufacturing system. Journal of Computing and Information Science in Engineering. 17: 021006.

ISO. 2002. Iso 19439, CIM system architecture-framework for enterprise modelling. Technical report, ISO.

Ji, X., He, G., Xu, J., and Guo, Y. 2016. Study on the mode of intelligent chemical industry based on cyber-physical system and its implementation. Advances in Engineering Software. 99: 18-26.

Jiang, P., Leng, J., Ding, K., Gu, P., and Koren, Y. 2016. Social manufacturing as a sustainable paradigm for mass individualization. Proceedings of the Institution of Mechanical Engineers, Part B: Journal of Engineering Manufacture. 230: 1961-1968.

Kang, S., Jeon, J., Kim, H.S., and Chun, I. 2016. Cps-based fault-tolerance method for smart factories. at-Automatisierungstechnik. 64: 750-757.

Koestler, A. 1968. The ghost in the machine. Oxford, UK: Macmillian.

Lee, E.A. 2008. Cyber physical systems: Design challenges. In $200811^{\text {th }}$ IEEE International Symposium on Object and Component-Oriented Real-Time Distributed Computing (ISORC), IEEE. p. 363-369.

Lee, R.M., Assante, M.J., and Conway, T. 2014. German steel mill cyber attack. Industrial Control Systems. 30, 2014.

Leitao, P., Karnouskos, S., Ribeiro, L., Lee, J. Strasser, T., and Colombo, A.W. 2016. Smart agents in industrial cyber-physical systems. Proceedings of the IEEE. 104: 1086-1101.

Lin, S.W., Miller, B., Durand, J., Joshi, R., and Didier, P. 2015. Industrial internet reference architecture. Technical report, Industrial Internet Consortium.

Liu, Y., and Xu, X. 2017. Industry 4.0 and cloud manufacturing: A comparative analysis. Journal of Manufacturing Science and Engineering. 139: 034701.

Lotterbach, S., and Peissner, M. 2005. Voice user interfaces in industrial environments. In GI Jahrestagung. p.592-596.

Louie, D.R., Eng, J.J., and Lam, T. 2015. Gait speed using powered robotic exoskeletons after spinal cord injury: a systematic review and correlational study. Journal of Neuroengineering and Rehabilitation. 12: 82.

Maier, A. 2014. Online passive learning of timed automata for cyber-physical production systems. In $12^{\text {th }}$ IEEE International Conference on Industrial Informatics (INDIN). IEEE. p. 60-66.

Makarov, O., Langmann, R., Nesterenko, S., and Frank, B. 2014. Problems of the time deterministic in applications for process control from the cloud. International Journal of Online Engineering. 10. 
Marini, A., and Bianchini, D. 2016. Big data as a service for monitoring cyber-physical production systems. In ECMS. p. 579-586.

Mathews, J.A. 1995. Holonic foundations of intelligent manufacturing systems. In 5th IFAC Symposium on Automated System Based on Human Skill, Joint Design of Technology and Organisation. p. 25-28.

McFarlane, D.C. 1995. Holonic manufacturing systems in continuous processing: concepts and control requirements. In Proceedings of ASI. 95: 273-282.

Mejás, A., Herrera, R.S., Márquez, M.A., Calderón, A.J., González, I., and Andújar, J.M. 2017. Easy handling of sensors and actuators over tcp/ip networks by open source hardware/software. Sensors. 17: 94.

MESA. 2008. Soa in manufacturing guidebook. Technical report, MESA International, IBM Corporation and Capgemini.

Mitra, S., and Acharya, T. 2007. Gesture recognition: A survey. IEEE Transactions on Systems, Man, and Cybernetics, Part C (Applications and Reviews). 37: 311324.

Monostori, L., KÃidÃir, B., Bauernhansl, T., Kondoh, S. Kumara, S., Reinhart, G., Sauer, O., Schuh, G., Sihn, W., and Ueda, K. 2016. Cyber-physical systems in manufacturing. CIRP Annals - Manufacturing Technology. 65: 621 - 641.

Moon, S. Kang, S., Jeon, J., and Chun, I. 2016. Simulation modeling of sewing process for evaluation of production schedule in smart factory. In 2016 International Conference on Industrial Engineering, Management Science and Application (ICIMSA). IEEE. p. 1-3.

Morariu, C., Morariu, O., Borangiu, T., and Raileanu, S. 2013. Manufacturing service bus integration model for highly flexible and scalable manufacturing systems. In T. Borangiu, A. Thomas, and D. Trentesaux, (eds.), Service Orientation in Holonic and Multi Agent Manufacturing and Robotics, pages 19-40. Berlin Heidelberg: Springer.

Morgan, J., and O'Donnell, G.A. 2017. Enabling a ubiquitous and cloud manufacturing foundation with field-level service-oriented architecture. International Journal of Computer Integrated Manufacturing. 30: 442-458.

Mourtzis, D., Vlachou, E., and Milas, N. 2016. Industrial big data as a result of iot adoption in manufacturing. Procedia CIRP. 55: 290-295.

Müller, R., Vette, M., Hörauf, L., Speicher, C., and Jatti, K. 2016. Concept and implementation of an agent-based control architecture for a cyber-physical assembly system. MATEC Web of Conferences. 42: 04003.

Neubauer, M., Krenn, F., Majoe, D., and Stary, C. 2017. Subject-orientation as design language for integration across organisational control layers. International Journal of Production Research. 55: 3644-3656.

Niggemann, O., and Frey, C. 2015. Data-driven anomaly detection in cyber-physical production systems. at-Automatisierungstechnik. 63: 821-832.

Olwal, A., Gustafsson, J., and Lindfors, C. 2008. Spatial augmented reality on industrial cnc-machines. In Electronic Imaging 2008, pages 680409-680409. International Society for Optics and Photonics.

OShaughnessy, D. 2008. Ínvited paper: Automatic speech recognition: History, methods and challenges. Pattern Recognition. 41: 2965 - 2979.

Oviatt, S., and Cohen, P. 2000. Perceptual user interfaces: Multimodal interfaces that process what comes naturally. Commun. ACM. 43:45-53.

Papazoglou, M.P., Traverso, P., Dustdar, S., and Leymann, F. 2007. Service-oriented computing: State of the art and research challenges. Computer. 40: 38-45.

Pasqualetti, F., Dörfler, F., and Bullo, F. 2013. Attack detection and identification in cyber-physical systems. IEEE Transactions on Automatic Control. 58: 27152729.

Penas, O., Plateaux, R., Patalano, S., and Hammadi, M. 2016. Multi-scale approach from mechatronic to cyber-physical systems for the design of manufacturing systems. Computers in Industry. 86: 52-69.

Pirvu, B.-C., Zamfirescu, C.-B., and Gorecky, D. 2016. Engineering insights from an anthropocentric cyber-physical system: A case study for an assembly station. Mechatronics. 34: 147-159.

Ploennigs, J., Clement, J., Wollschlaeger, B., and Kabitzsch, K. 2016. Semantic models for physical processes in cps at the example of occupant thermal comfort. In 
2016 IEEE $25^{\text {th }}$ International Symposium on Industrial Electronics (ISIE). IEEE. p. $1061-1066$.

Posada, J., Toro, C., Barandiaran, I., Oyarzun, D., Stricker, D., DeAmicis, R., Pinto, E.B., Eisert, P., Döllner, J., and Vallarino, I. 2015. Visual computing as a key enabling technology for industrie 4.0 and industrial internet. IEEE computer graphics and applications. 35: 26-40.

Puhm, A., Mahmood, A., Bigler, T., and Kerö, N. 2016. Synchronizing an ieee 1588 slave clock over both paths of a redundant ethernet system. In 2016 IEEE International Symposium on Precision Clock Synchronization for Measurement, Control, and Communication (ISPCS). IEEE. p. 1-6.

Pujo, P., Ounnar, F., Power, D., and Khader, S. 2016. Wireless holon network for job shop isoarchic control. Computers in Industry 83: 12-27.

Puttonen, J., Lobov, A., Cavia Soto, M.A., and Martinez Lastra, J.L. 2015. Planningbased semantic web service composition in factory automation. Advanced Engineering Informatics. 29: 1041-1054.

Quintanilla, F.G., Cardin, O., L'Anton, A., and Castagna, P. 2016. Implementation framework for cloud-based holonic control of cyber-physical production systems. In 14th International Conference on Industrial Informatics (INDIN), IEEE. p. 316-321.

Raskar, R., Welch, G., and Fuchs, H. 1998. Spatially augmented reality. In First IEEE Workshop on Augmented Reality (IWAR ${ }^{\mathrm{TM}} 98$ ). p. 11-20.

Rojas, R.A., Rauch, E., Vidoni, R., and Matt, D.T. 2017. Enabling connectivity of cyber physical production systems: A conceptual framework. Procedia Manufacturing. 11: 822-829.

Rojas, R.A. and Rauch, E. 2019. From a literature review to a conceptual framework of enablers for smart manufacturing control. International Journal of Advanced Manufacturing Technology. 104: 517-533.

Saldivar, A.A.F., Li, Y., Chen, W., Zhan, Z., Zhang, J., and Chen, L.Y. 2015. Industry 4.0 with cyber-physical integration: a design and manufacture perspective. In $21^{\text {st }}$ international conference on Automation and computing (icac). IEEE. p. $1-6$.

Sansoni, S., Trebeschi, M., and Docchio, F. 2009. State-of-the-art and applications of 3d imaging sensors in industry, cultural heritage, medicine, and criminal investigation. Sensors. 9: 568-601.

Sauer. O. 2014. Developments and trends in shopfloor-related ict systems. In 2014 IEEE International Conference on Industrial Engineering and Engineering Management (IEEM). IEEE. p. 1352-1356.

Schmidt, N., Lüder, A., Rosendahl, R., Ryashentseva, D., Foehr, M., and Vollmar, J. 2015. Surveying integration approaches for relevance in cyber physical production systems. In 2015 IEEE $20^{\text {th }}$ Conference on Emerging Technologies \& Factory Automation (ETFA). IEEE. p. 1-8.

Schmitt, R.H., Peterek, M., Morse, E., Knapp, W., Galetto, M., Härtig, F., Goch, G., Hughes, B., Forbes, A., and Estler, W.T. 2016. Advances in large-scale metrology-review and future trends. CIRP Annals-Manufacturing Technology. 65: 643-665.

Schlechtendahl, J., Keinert, M., Kretschmer, F., Lechler, A., and Verl, V. 2015. Making existing production systems industry 4.0-ready. Production Engineering. 9: 143148.

Schleipen, M., Lüder, A., Sauer, O., Flatt, H., and Jasperneite, J. 2015. Requirements and concept for plug-and-work. at-Automatisierungstechnik. 63: 801-820.

Shields, B.L., Main, J.A., Peterson, S.W., and Strauss, A.M. 1997. An anthropomorphic hand exoskeleton to prevent astronaut hand fatigue during extravehicular activities. IEEE transactions on systems, man, and cybernetics-part A: systems and humans. 27: 668-673.

Skarlat, O., Borkowski, M., and Schulte, S. 2016. Towards a methodology and instrumentation toolset for cloud manufacturing. In 2016 1st International Workshop on Cyber-Physical Production Systems (CPPS), IEEE. p. 1-4. 
Slay, J., and Miller, M. 2007. Lessons learned from the maroochy water breach. In International Conference on Critical Infrastructure Protection, Springer. p. 7382.

Tao, F., Zhang, L., Venkatesh, V.C., Luo, Y., and Cheng, Y. 2011. Cloud manufacturing: a computing and service-oriented manufacturing model. Proceedings of the Institution of Mechanical Engineers, Part B: Journal of Engineering Manufacture. 225: $1969-1976$.

Tao, F., Cheng, J., Cheng, Y., Gu, S., Zheng, T., and Yang, H. 2017. Sdmsim: a manufacturing service supply-demand matching simulator under cloud environment. Robotics and computer-integrated manufacturing. 45: 34-46.

Taylor, R., Jensen, P., Whitcomb, L., Barnes, A., Kumar, R., Stoianovici, D., Gupta, P., Wang, Z.X., Dejuan, E., and Kavoussi, L. 1999. A steady-hand robotic system for microsurgical augmentation. The International Journal of Robotics Research. 18: $1201-1210$.

Teixeira, A., Pérez, S., Sandberg, H., and Johansson, K.H. 2012. Attack models and scenarios for networked control systems. In Proceedings of the 1st international conference on High Confidence Networked Systems. ACM. p. 55-64.

Tharumarajah, A. 1996. Comparison of the bionic, fractal and holonic manufacturing system concepts. International Journal of Computer Integrated Manufacturing. 9: 217-226.

Thramboulidis, K., and Christoulakis F. 2016. Uml4IOT - a uml-based approach to exploit iot in cyber-physical manufacturing systems. Computers in Industry. 82: 259-272.

Tranfield, D., Denyer, D., and Smart, P. 2003. Towards a methodology for developing evidence-informed management knowledge by means of systematic review. British Journal of Management. 14: 207-222.

Triesch, J., and von der Malsburg, C. 1998. Robotic gesture recognition, pages 233244. Berlin, Heidelberg: Springer.

Ullrich, J., Voyiatzis, A.G. and Weippl, E.R. 2016. Secure cyber-physical production systems: Solid steps towards realization. In 2016 1st International Workshop on Cyber-Physical Production Systems (CPPS), IEEE. p. 1-4.

Vaquero, L.M., Rodero-Merino, L., Caceres, J., and Lindner, M. 2008. A break in the clouds: towards a cloud definition. ACM SIGCOMM Computer Communication Review. 39: 50-55.

Verl, A., Lechler, A., and Schlechtendahl, J. 2012. Glocalized cyber physical production systems. Production Engineering. 6: 643-649.

Vernadat, F.B. 2007. Interoperable enterprise systems: Principles, concepts, and methods. Annual Reviews in Control. 31: 137 - 145.

Vogel-Heuser, B., Lee, J., and Leitão, P. 2015. Agents enabling cyber-physical production systems. at-Automatisierungstechnik. 63: 777-789.

Waitzinger, S., Ohlhausen, P., and Spath, D. 2015. The industrial internet. business models as challenges for innovations. In Proceedings of the International Conference on Production Research (ICPR) Manila, Philippines.

Wang, L., Törngren, M., and Onori, M. 2015. Current status and advancement of cyberphysical systems in manufacturing. Journal of Manufacturing Systems. 37: 517527.

Wang, L., and Haghighi, A. 2016. Combined strength of holons, agents and function blocks in cyber-physical systems. Journal of Manufacturing Systems. 40: 25-34.

Wang, S., Wan, J., Imran, M., Li, D., and Zhang, C. 2016. Cloud-based smart manufacturing for personalized candy packing application. The Journal of Supercomputing. 74: 4339-4357.

Weckenmann, A., and Hartmann, W. 2015. A model-and simulation-based approach for tolerancing and verifying the functional capability of micro/nano-structured workpieces. Measurement. 76: 70-79.

Weichhart, G., Guédria, W., and Naudet, Y. 2016. Supporting interoperability in complex adaptive enterprise systems: A domain specific language approach. Data \& Knowledge Engineering. 105: 90-106.

Weinberger, M., Bilgeri, D., and Fleisch, E. 2016. Iot business models in an industrial context. at-Automatisierungstechnik. 64: 699-706. 
Weiss, G. 1999. Multiagent systems: a modern approach to distributed artificial intelligence. Boston: MIT press.

Windmann, S., Maier, A., Niggemann, O., Frey, C., Bernardi, A., Gu, Y., Pfrommer, H., Steckel, T., Krüger, M., and Kraus, R. 2015. Big data analysis of manufacturing processes. In Journal of Physics: Conference Series. 659: 012055. IOP Publishing.

Wooldridge, M., and Jennings, N.R. 1995. Intelligent agents: Theory and practice. The Knowledge Engineering Review. 10: 115-152.

Wu, G., Sun, J., and Chen, J. 2016. A survey on the security of cyber-physical systems. Control Theory and Technology. 14: 2-10.

Yampolskiy, M., Horvath, P., Koutsoukos, X.D., Xue, Y., and Sztipanovits, J. 2013. Taxonomy for description of cross-domain attacks on cps. In Proceedings of the $2^{\text {nd }}$ ACM international conference on High confidence networked systems. ACM. p. $135-142$.

Yen, C.-T., Liu, Y.-C., Lin, C.-C., Kao, C.-C., Wang, W.-B., and Hsu, Y.-R. 2014. Advanced manufacturing solution to industry 4.0 trend through sensing network and cloud computing technologies. In 2014 IEEE International Conference on Automation Science and Engineering (CASE). IEEE. p. 1150-1152.

Yoon, S.C., and Suh, S.H. 2016. Manufacturing information bus from the perspective of cyber physical manufacturing system (cpms). IFAC-PapersOnLine. 49: 103-108.

Xu, Y., Song, R., Korba, L., Wang, L. 2005. Weiming Shen, and Sherman Lang. Distributed device networks with security constraints. IEEE Transactions on Industrial Informatics. 1: 217-225.

$\mathrm{Xu}, \mathrm{X} .22012$. From cloud computing to cloud manufacturing. Robotics and ComputerIntegrated Manufacturing. 28: 75-86.

Zheng, B., Deng, P., Anguluri, R., Zhu, Q., and Pasqualetti, F. 2016. Cross-layer codesign for secure cyber-physical systems. IEEE Transactions on ComputerAided Design of Integrated Circuits and Systems. 35: 699-711.

Zhou, K., Liu, T., and Zhou, L. 2015. Industry 4.0: Towards future industrial opportunities and challenges. In 2015 12th International Conference on Fuzzy Systems and Knowledge Discovery (FSKD). IEEE. p. 2147-2152.

Zhou, K., Liu, T., and Liang, L. 2016. From cyber-physical systems to industry 4.0: make future manufacturing become possible. International Journal of Manufacturing Research. 11: 167-188.

Zimmermann, H. 1980. Osi reference model-the iso model of architecture for open systems interconnection. IEEE Transactions on communications. 28: 425-432.

Zolotová, I., Bundzel, M., and Lojka, T. 2015. Industry IoT Gateway for Cloud Connectivity. Cham: Springer. p. 59-66. 